\title{
Neumann Resonances in Linear Elasticity for an Arbitrary Body
}

\author{
P. Stefanov ${ }^{1, \star, \star \star}$, G. Vodev ${ }^{2}$ \\ 1 Institute of Mathematics, Bulgarian Academy of Sciences, Acad. G. Bontchev str., bl. 8, 1113 \\ Sofia, Bulgaria \\ ${ }^{2}$ Université de Rennes 1, IRMAR, URA 305 du CNRS, Campus de Beaulieu, F-35042 Rennes \\ Cedex, France
}

Received: 6 December 1994

\begin{abstract}
We study resonances (scattering poles) associated to the elasticity operator in the exterior of an arbitrary obstacle in $\mathbf{R}^{3}$ with Neumann boundary conditions. We prove that there exists a sequence of resonances tending rapidly to the real axis.
\end{abstract}

\section{Introduction}

Let $\mathcal{O} \subset \mathbf{R}^{3}$ be a compact set with $C^{\infty}$-smooth boundary $\Gamma$ and connected complement $\Omega=\mathbf{R}^{3} \backslash \mathcal{O}$. Denote by $\Delta_{e}$ the elasticity operator

$$
\Delta_{e} v=\mu_{0} \Delta v+\left(\lambda_{0}+\mu_{0}\right) \nabla(\nabla \cdot v),
$$

$v={ }^{t}\left(v_{1}, v_{2}, v_{3}\right)$. Here $\lambda_{0}, \mu_{0}$ are the Lamé constants and we assume that

$$
\mu_{0}>0, \quad 3 \lambda_{0}+2 \mu_{0}>0 .
$$

Consider $A_{e}$ in $\Omega$ with Neumann boundary conditions on $\Gamma$,

$$
\left.\sum_{j=1}^{3} \sigma_{i j}(v) v_{J}\right|_{\Gamma}=0, \quad i=1,2,3,
$$

where $\sigma_{i j}(v)=\lambda_{0} \nabla \cdot v \delta_{i j}+\mu_{0}\left(\frac{\partial v_{l}}{\partial x_{J}}+\frac{\partial v_{j}}{\partial x_{l}}\right)$ is the stress tensor, $v$ is the outer normal to $\Gamma$. Denote by $L$ the self-adjoint realization of $-\Delta_{e}$ in $\Omega$ with Neumann boundary conditions on $\Gamma$. As usual we define resonances as the poles of the meromorphic continuation of the cut-off resolvent $R_{\chi}(\lambda)=\chi\left(L-\lambda^{2}\right)^{-1} \chi$ from $\operatorname{Im} \lambda<0$ to the whole complex plane $\mathbf{C}, \chi \in C_{0}^{\infty}$ being a cut-off function equal to 1 near $\Gamma$. So we accept the convention that the resonances lie in the upper half-plane.

If one considers the Laplacian with Dirichlet or Neumann boundary conditions, then it is well known that for convex or more generally for non-trapping obstacles

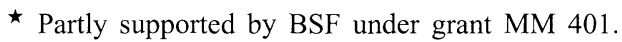

$\star \star$ Current address: Department of Mathematics, University of British Columbia, Vancouver, B.C., V6T 1Y4, Canada. 
the resonances lie above logarithmic curves of the type $\operatorname{Im} \lambda=C_{1} \ln \operatorname{Re} \lambda-C_{2}$, $C_{1}>0$. There are several special examples of trapping obstacles [I1, I2, I3, G] with resonances tending to the real axis or with a strip of the kind $0<\operatorname{Im} \lambda<C_{0}$ containing infinitely many resonances. An important open problem in this direction is to prove or reject the Modified Lax and Phillips Conjecture-for any trapping obstacle there is a strip $0<\operatorname{Im} \hat{i}<C_{0}$ containing infinitely many resonances.

In [SV2] the authors proved that for the elasticity operator $L$ with Neumann boundary conditions there exists a sequence of resonances tending rapidly to the real axis provided that the obstacle $\mathcal{O}$ is strictly convex. Moreover, below any logarithmic curve $\operatorname{Im} \lambda=C_{1} \ln |\lambda|-C_{2}$ there are no other resonances except possibly a finite number. The reason for the existence of almost real resonances are the Rayleigh waves which is a typical phenomenon for the elasticity operator with Neumann boundary conditions. As proven by Taylor [T] (see also [Y]) there are three types of rays for $L$ that carry singularities. The first two types are classical rays reflecting at the boundary according to the laws of geometrical optics and the singularities propagate along them with speeds $c_{1}=\sqrt{\mu_{0}}, c_{2}=\sqrt{\lambda_{0}+2 \mu_{0}}$. The third type of trajectories lie on the boundary and singularities propagate along them with a slower propagation speed $c_{R}>0$ (the Rayleigh speed). Thus any obstacle is trapping for $L$ from the point of view of propagation of singularities and one might expect resonances close to the real axis. The proof in [SV2] is based on a construction of a microlocal parametrix of the corresponding Neumann operator in all of the 5 zones (hyperbolic, mixed, elliptic and two glancing ones) using the calculus of $\Psi$ DO-s and FIO-s with large parameter (sce e.g. [G]). It turns out that the parametrix is elliptic in the first two zones, can be represented microlocally as a hypoelliptic operator in $L_{2 / 3,0}^{0,1}$ conjugated with an elliptic FIO in the glancing zone while in the elliptic zone has a characteristic variety of the form $\Sigma=\left\{\zeta \in T^{*} \Gamma ; c_{R}\|\zeta\|=1\right\}$. Therefore, the parametrix is microlocally invertible outside $\Sigma$, which is essential for the proof of the pole-free domain, while the proof of the existence of almost real resonances is based on an application of the Phragmén-Lindelöf principle.

In this paper we show the existence of a sequence of resonances of $L$ tending to the real axis for an arbitrary obstacle $\mathcal{O}$. Of course, one can no longer expect a pole-free logarithmic zone as in the case of a strictly convex obstacle [SV2] because there might be resonances near the real axis or more generally in any logarithmic region generated by classical trapped rays. Our main result is the following theorem.

Theorem 1. There exist two infinite sequences $\left\{\lambda_{j}\right\},\left\{-\bar{\lambda}_{j}\right\}$ of distinct resonances of the elasticity operator $L$, such that

$$
0<\operatorname{Im} \lambda_{j} \leqq C_{N}\left|\lambda_{j}\right|^{-N} \quad \text { for any } N>0 .
$$

The proof of Theorem 1 suggests that the reason for the existence of these resonances are the Rayleigh waves. In particular, it provides another proof of Kawashita's result $[\mathrm{K}]$ that the elastic wave equation with Neumann boundary conditions does not possess the exponential local energy decay property.

To prove Theorem 1 it suffices to show that for any integer $N \geqq 1$ there are infinitely many resonances in $\left\{\lambda \in \mathbf{C}: \operatorname{Im} \lambda \leqq|\lambda|^{-N},|\operatorname{Re} \lambda| \geqq 1\right\}$. Then, the assumption that there are finitely many resonances in this region would lead to polynomial a priori estimates on $\mathscr{N}^{-1}(\lambda), \mathscr{N}(\lambda)$ being the Neumann operator on $\Gamma$, in a smaller region near the real axis. The final step is to show that these a priori estimates cannot hold because the parametrix of the Neumann operator fails to be elliptic at $\Sigma$. To do so, we use the calculus of $\Psi$ DO-s and FIO-s with large 
parameter as presented in $[\mathrm{G}]$ to construct a parametrix of the Neumann operator in the elliptic zone. Note that this is possible despite the fact that $\mathcal{O}$ is not necessarily convex. In fact, it is sufficient to construct the parametrix in a neighborhood of the characteristic variety $\Sigma$. We take a finite number $2 m+1$ of terms in the asymptotic expansion of the corresponding amplitude in order to get a parametrix $N_{2 m-1}(\lambda)$, such that $\lambda^{2 m-1} N_{2 m-1}(\lambda)$ is analytic in $\lambda$. Then we extend $\lambda^{2 m-1} N_{2 m-1}(\lambda)$ as an elliptic $\Psi$ DO with large parameter $\lambda_{1}=\operatorname{Re} \lambda$ globally, thus obtaining a $\Psi$ DO $P(\lambda)$ which is an entire function of $\lambda$. Applying the Phragmén-Lindelöf principle, we show that $P\left(\lambda_{i}\right)$ has "zeros," i.e. we have $P\left(\lambda_{j}\right) f_{j}=0$ with some $\lambda_{j}$ in a logarithmic domain and $\left\|f_{j}\right\|=1$. Next we show that $\lambda_{j}$ are asymptotic zeros of $N_{2 m-1}(\lambda)$, i.e. $N_{2 m-1}\left(\lambda_{j}\right) f_{j}=O\left(\left|\lambda_{j}\right|^{-\infty}\right)$, as well as $\operatorname{Im} \lambda_{j}=O\left(\left|\lambda_{j}\right|^{-2 m+2}\right)$. Since $N_{2 m-1}$ is a parametrix of the Neumann operator $\mathcal{N}$, for $m$ sufficiently large this turns out to be enough to get the desired contradiction.

\section{Some A Priori Estimates on the Resolvent}

The purpose of this section is to prove the following a priori estimate of the cut-off resolvent which is crucial for our proof of Theorem 1 .

Proposition 1. Assume that $R_{\chi}(\lambda)$ is analytic in $\left\{\hat{\lambda} \in \mathbf{C} ; \operatorname{Im} \lambda \leqq|\lambda|^{-N},|\operatorname{Re} \lambda| \geqq C\right\}$ with some $C>0$ and integer $N>0$. Then

$$
\left\|R_{\%}(\lambda)\right\|_{\mathcal{L}\left(L^{2} ; H^{2}\right)} \leqq C_{1}|\lambda|^{N+7} \text { for } \quad|\operatorname{Im} \lambda| \leqq|\lambda|^{-N-6}, \quad|\operatorname{Re} \lambda| \geqq C_{2},
$$

with some constants $C_{1}, C_{2}>0$.

Remark 1. It is easy to see from the proof that a similar statement holds in any odd-dimensional space as well and for compactly supported perturbations of the Laplacian. The proof however does not work if the space dimension is even.

Remark 2. As an immediate consequence of Proposition 1 we get that the existence of real quasimodes implies the existence of resonances $\left\{\lambda_{j}\right\}_{j=1}^{\infty}$ with $\operatorname{Im} \lambda_{j}=$ $O\left(\left|\lambda_{j}\right|^{-\infty}\right)$. The advantage of this conclusion is that, as shown in [P] (see also $[\mathrm{CP}, \mathrm{L}]$ ), if there exists an elliptic broken periodic ray (with Poincaré map satisfying some technical conditions), one can construct real quasimodes $k_{j} \rightarrow+\infty$ of the Dirichlet Laplacian in an exterior domain $\Omega \subset \mathbf{R}^{n}, n \geqq 3$ odd, with $C^{\infty}$ boundary $\Gamma$, i.e. there exist uniformly compactly supported functions $u_{j},\left\|u_{j}\right\|_{L^{2}}=1$, such that

$$
\begin{cases}\left(\Delta+k_{j}^{2}\right) u_{j}=O\left(k_{j}^{-\infty}\right) & \text { in } \Omega, \\ u_{j}=O\left(k_{j}^{-\infty}\right) & \text { on } \Gamma .\end{cases}
$$

The proof of Proposition 1 is based on the next two lemmae.

Lemma 1. Assume that $f(z)$ is analytic in $\left\{z \in \mathbf{C} ; \operatorname{Im} z<C_{1}|z|^{-N},|\operatorname{Re} z|>C_{2}\right\}$ and $|f(z)| \leqq C_{3} e^{C_{3}|z|^{n}}$ with some positive constants $C_{1}, C_{2}, C_{3}$, and integers $n, N \geqq$ 0 . Assume moreover that $|f(z)| \leqq C_{4}|z|^{m} /|\operatorname{Im} z|$ for $-1<\operatorname{Im} z<0,|z|>1$ with some $C_{4}>0$ and integer $m \geqq 0$. Then

$$
|f(z)| \leqq C_{5}|z|^{m+n+N+2} \text { for }|\operatorname{Im} z| \leqq|z|^{-n-N-2}, \quad|\operatorname{Re} z| \geqq C_{5},
$$

with some constant $C_{5}>0$. 
Proof. Without loss of generality we can assume that $\operatorname{Re} z \geqq C_{2}$. Set

$$
u(z)=\exp \left\{i z^{s}\right\}=\exp \left\{i(\operatorname{Re} z)^{s}-s(\operatorname{Re} z)^{s-1} \operatorname{Im} z+\cdots\right\},
$$

$s$ being an integer to be chosen later on. On $\gamma_{+}:=\left\{z \in \mathbf{C} ; \operatorname{Im} z=C_{1}|z|^{-N}\right.$, $\left.\operatorname{Re} z \geqq C_{2}\right\}$ we have for $g:=f u$,

$$
\begin{aligned}
|g(z)| & \leqq C_{3} e^{C_{3}|z|^{n}} \exp \left\{-s(\operatorname{Re} z)^{s-1} \operatorname{Im} z+C_{s}(\operatorname{Re} z)^{s-3}(\operatorname{Im} z)^{3}-\cdots\right\} \\
& =C_{3} e^{C_{3}|z|^{n}} \exp \left\{-C_{1} s \frac{(\operatorname{Re} z)^{s-1}}{|z|^{N}}+C_{s}^{\prime} \frac{(\operatorname{Re} z)^{s-3}}{|z|^{3 N}}-\cdots\right\} \\
& \leqq C_{3} e^{C_{3}|z|^{n}} \exp \left\{-\frac{1}{2} C_{1} s \frac{(\operatorname{Re} z)^{s-1}}{|z|^{N}}\right\}
\end{aligned}
$$

for $|z|$ sufficiently large. Therefore, if $s>n+N+1$, we have

$$
|g(z)| \leqq C \quad \text { for } z \in \gamma_{+} .
$$

Set $\gamma_{-}=\left\{z \in \mathbf{C} ;-\operatorname{Im} z=|z|^{-s}, \operatorname{Re} z \geqq C_{2}\right\}$. On $\gamma_{-}$we have

$$
|g(z)| \leqq \frac{C_{4}|z|^{m}}{|\operatorname{Im} z|} \exp \left\{s(\operatorname{Re} z)^{s-1}|\operatorname{Im} z|-C_{s}(\operatorname{Re} z)^{s-3}|\operatorname{Im} z|^{3}+\cdots\right\} \leqq C|z|^{m+s}
$$

We get from (4), (5) that $z^{-m-s} g(z)$ is uniformly bounded on the boundary of the domain between the curves $\gamma_{+}, \gamma_{-}$and $\operatorname{Re} z=C_{2}^{\prime}$ with $C_{2}^{\prime}>C_{2}$ sufficiently large. Moreover $z^{-m-s} g(z)$ satisfies an a priori exponential estimate in the interior. An application of the Phragmén-Lindelöf principle implies that $z^{-m-s} g(z)$ is uniformly bounded in the interior of that domain as well and therefore

$$
|f(z)| \leqq C|z|^{m+s} \exp \left\{s(\operatorname{Re} z)^{s-1} \operatorname{Im} z-C_{s}(\operatorname{Re} z)^{s-3}(\operatorname{Im} z)^{3}+\cdots\right\} \leqq C_{5}|z|^{m+s},
$$

provided that $|\operatorname{Im} z| \leqq 1 /(\operatorname{Re} z)^{s}$. Now it suffices to pick $s=N+n+2$ in order to complete the proof of the lemma.

We would like to apply this lemma to the operator-valued function $R_{\chi}(\lambda)$. To this end we need the following a priori estimate (compare with [SV2, Proposition 5.2]).

Lemma 2. Assume that $R_{\chi}(\lambda)$ is analytic in $D_{C_{1}, C_{2}}=\left\{\lambda \in \mathbf{C} ;|\operatorname{Im} \lambda|<C_{1}|\lambda|^{-N}\right.$, $\left.|\operatorname{Re} \lambda|>C_{2}\right\}$ with some $C_{1}>0, C_{2}>0, N>0$. Then for any $C_{1}^{\prime}<C_{1}, C_{2}^{\prime}>C_{2}$ we have

$$
\left\|R_{\gamma}(\lambda)\right\|_{\mathcal{L}\left(L^{2} ; H^{2}\right)} \leqq C e^{C|\lambda|^{4}}
$$

with some $C>0$ in $D_{C_{1}^{\prime}, C_{2}^{\prime}}=\left\{\lambda \in \mathbf{C} ;|\operatorname{Im} \lambda|<C_{1}^{\prime}|\lambda|^{-N},|\operatorname{Re} \lambda|>C_{2}^{\prime}\right\}$.

Proof. This is a refinement of Proposition 5.2 in [SV2] (see also Lemma 3 in the present paper) and we refer to [SV2] for more details. As in the above cited paper we can find an entire function $h(\lambda)$ of order 3 , such that in

we have

$$
V=\mathbf{C} \backslash \bigcup_{j}\left\{\lambda ;\left|\lambda-z_{J}\right|<\left|z_{J}\right|^{-5-N}\right\}
$$

$$
\left\|R_{\chi}(\lambda)\right\|_{\mathcal{L}\left(L^{2} ; H^{2}\right)} \leqq C e^{C|\hat{\lambda}|^{4}} \quad \text { for } \lambda \in V
$$


where $\left\{z_{\jmath}\right\}_{j=1}^{\infty}$ are the zeros of $h(\lambda)$. Let us observe that $\mathbf{C} \backslash V=\bigcup_{k=1}^{\infty} U_{k}$, where $U_{k}$ are disjoint connected sets and each $U_{k}$ is a union of a finite number of disks, because the series $\sum_{j=1}^{\infty}\left|z_{j}\right|^{-4}$ converges and hence so does $\sum_{j=1}^{\infty}\left|z_{j}\right|^{-5-N}$. Clearly, for each $k$, diam $U_{k}<2 M$, where $M:=\sum\left|z_{j}\right|^{-4}$, which implies

Therefore,

$$
\sum_{\left|z_{j}\right|>A}\left|z_{j}\right|^{-N-5} \leqq M A^{-N-1}
$$

$$
\operatorname{diam} U_{k}<2 M\left[\min \left\{|\lambda| ; \lambda \in U_{k}\right\}\right]^{-N-1} \leqq 2 M(|\lambda|-2 M)^{-N-1}
$$

for each $\lambda \in U_{k}$, and $k$ sufficiently large. Fix $C_{1}^{\prime}<C_{1}, C_{2}^{\prime}>C_{2}$ and set $K=\{k \in \mathbf{N}$; $\left.D_{C_{1}^{\prime}, C_{2}^{\prime}} \cap U_{k} \neq \emptyset\right\}$. For large $k \in K$ we have $U_{k} \subset D_{C_{1}, C_{2}}$ because of (8). Since (7) holds on $\partial U_{k}$ we can apply the maximum principle to conclude that (7) holds in $U_{k}$ as well with some other constant $C>0$ for large $k$. Thus (7) holds in the entire $D_{C_{1}^{\prime}, C_{2}^{\prime}}$ except perhaps in a bounded set.

Proof of Proposition 1. We have by the above lemma that the operator-valued function $R_{\lambda}(\lambda): L^{2} \rightarrow H^{2}$ satisfies the first assumption of Lemma 1 with $n=4$. On the other hand, it is clear that in the lower half-plane $R_{\gamma}$ satisfies the estimate

which easily yields

$$
\left\|R_{\chi}(\lambda)\right\|_{\mathcal{L}\left(L^{2}\right)} \leqq \frac{C}{|\operatorname{Im} \lambda||\lambda|},
$$

$$
\left\|R_{\chi}(\lambda)\right\|_{\mathcal{L}\left(L^{2} ; H^{2}\right)} \leqq \frac{C^{\prime}|\lambda|}{|\operatorname{Im} \lambda|} \text { for } \operatorname{Im} \lambda<0 .
$$

Thus $R_{\chi}(\lambda): L^{2} \rightarrow H^{2}$ satisfies the second assumption of Lemma 1 as well with $m=1$. Now Proposition 1 follows from Lemma 1 at once.

Let us define the Neumann operator $\mathscr{N}(\lambda)$ by the formula

$$
\mathscr{N}(\lambda):\left.H^{s}(\Gamma) \ni f \mapsto \sum_{j=1}^{3} \boldsymbol{\sigma}_{j}(v) v_{j}\right|_{\Gamma} \in H^{s-1}(\Gamma), \quad s \geqq \frac{3}{2},
$$

where $\boldsymbol{\sigma}_{j}={ }^{t}\left(\sigma_{1 j}, \sigma_{2 j}, \sigma_{3 j}\right), \sigma_{i j}$ is the stress tensor (see (2)) and $v$ solves the following problem:

$$
\begin{cases}\left(\Delta_{e}+\lambda^{2}\right) v=0 & \text { in } \Omega \\ v=f & \text { on } \Gamma \\ v \text { - outgoing } . & \end{cases}
$$

As $\mathscr{N}^{-1}(\lambda)$ can be easily expressed in terms of $R_{\gamma}(\lambda)$ (see [SV1]), the assumption that $R_{\gamma}(\lambda)$ is holomorphic in $\left\{\lambda \in \mathbf{C}: \operatorname{Im} \lambda \leqq|\lambda|^{-N},|\operatorname{Re} \lambda| \geqq C\right\}$ implies that so is $\mathscr{N}^{-1}(\lambda)$ and moreover, by Proposition 1 ,

$$
\left\|\mathscr{N}^{-1}(\lambda)\right\|_{\mathcal{L}\left(H^{1 / 2}, H^{3 / 2}\right)} \leqq C|\lambda|^{N+9} \text { for }|\operatorname{Im} \lambda| \leqq|\lambda|^{-N-6},|\operatorname{Re} \lambda| \geqq C^{\prime}
$$

In the rest of the paper we will find a contradiction to (10).

\section{Parametrix for the Neumann Operator}

We will recall briefly the construction of the parametrix of (9) in the elliptic zone (see $[\mathrm{SV} 2, \mathrm{CP}]$ ). We will use the calculus of $\Psi \mathrm{DO}-\mathrm{s}$ and FIO-s with large parameter 
as developed in [G]. We choose $\lambda$ to be the large parameter and we suppose that

$$
\lambda \in \Lambda_{C_{1}, C_{2}}:=\left\{\lambda \in \mathbf{C} ;\left|\lambda_{2}\right|<C_{1} \ln \lambda_{1}, \lambda_{1}>C_{2}\right\},
$$

where $\lambda_{1}=\operatorname{Re} \lambda, \lambda_{2}=\operatorname{Im} \lambda, C_{1}>0, C_{2}>0$.

Given an open set $X$ in $\mathbf{R}^{n}$ denote by $\tilde{C}^{\infty}(X)$ the space of all functions $u(x, \lambda)$, $\lambda \in \Lambda$ such that $u(\cdot, \lambda) \in C^{\infty}(X)$ and $p(u(\cdot, \lambda))=O\left(|\lambda|^{-\infty}\right)$ for all seminorms $p$ in $C^{\infty}(X)$. In a similar way we define $\tilde{C}^{\infty}(K), K$ being a compact, $\tilde{C}_{0}^{\infty}(X)$ and $\tilde{\mathscr{D}}^{\prime}(X)$.

Given two open sets $X, Y$ in $\mathbf{R}^{n}$, for $m, k \in \mathbf{R}, \rho, \delta \in[0,1)$ we define (see [G, Def. A.I.2]) the class $S_{\rho, \delta}^{m, k}(X \times Y)$ to be the set of all $a(x, y, \eta, \hat{\imath}) \in C^{\infty}(X \times$ $Y \times \mathbf{R}^{n}$ ), such that for any compact $K \subset \subset X \times Y$, all $\alpha, \beta, \gamma \in \mathbf{Z}^{n}, \lambda \in \Lambda$ we have

$$
\left|\partial_{x}^{\alpha} \partial_{y}^{\beta} \partial_{\eta}^{i} a\right| \leqq C_{\gamma, \beta, \gamma, K}|\lambda|^{k+\rho|;|+\delta|x+\beta|}(1+|\eta|)^{m-|\gamma|}
$$

If $X=Y$, we set $S_{\rho, \delta}^{m, k}(X)=S_{\rho, \delta}^{m, k}(X \times X)$. Given $a \in S_{\rho, \delta}^{m, k}(X \times Y)$, denote by $\operatorname{Op}(a)$ (or $\mathrm{Op}_{j}(a)$ ) the operator

$$
(\operatorname{Op}(a) u)(x, \lambda)=\left(\frac{\lambda}{2 \pi}\right)^{n} \iint e^{i \lambda(x-y) \cdot \eta} a(x, y, \eta, \lambda) u(y, \lambda) d y d \eta .
$$

We have well-defined operators in the case where $a$ has bounded support in $\eta$ and $\lambda \in \Lambda$ or if $\eta$ is unbounded on $\operatorname{supp} a$, but $\lambda$ is real. We refer to [G] (see also [SV2]) for more details, as well as for a definition and properties of elliptic $\Psi D O-\mathrm{s}$ with large parameter, wave front set $\widehat{\mathrm{WF}}(f)$, etc.

Let us recall [SV2] that operators of the form $\mathrm{Op}_{j}(a)$ can be represented as $\Psi$ DO-s with large parameter $\lambda_{1}=\operatorname{Re} \lambda$, provided that $|\eta|$ is bounded on $\operatorname{supp} a$. In other words, $\mathrm{Op}_{i}(a)=\mathrm{Op}_{i_{1}}(\tilde{a})$, where

$$
\tilde{a}(x, y, \eta, \lambda)=\left(1+i \lambda_{2} / \lambda_{1}\right)^{n} e^{-\lambda_{2}(x-y)} \cdot \eta a(x, y, \eta, \lambda) .
$$

One can regard here $\lambda_{2} / \lambda_{1} \in\left[-C_{1}, C_{1}\right]$ as an additional parameter. Assuming $\lambda \in \Lambda_{C_{1}, C_{2}}$, we get that $a \in S_{\rho, \delta}^{0, k}$ implies $\tilde{a} \in S_{\rho+\varepsilon, d+\varepsilon}^{0, k+N}$ for any $\varepsilon>0$. This follows from the fact that $\left|e^{-i_{2}(x-y)} \cdot \eta\right| \leqq|\lambda|^{N}$ with a fixed $N>0$ and $\left|\lambda_{2}\right| \leqq C_{\varepsilon}|\lambda|^{\varepsilon}$ for any $\varepsilon>0$. Therefore, $\tilde{a}$ is an amplitude. Using [G, Pr. A.I.4, Pr. A.I.5] we can calculate the symbol of $\tilde{a}$ (depending only on $x, \eta, \lambda$ ) and we find that actually $\tilde{a} \in S_{\rho, d}^{0, k}$ and the principal symbol is $\left.a\right|_{y=x}-\left.i\left(\lambda_{2} / \lambda_{1}\right) \eta \cdot \nabla_{\eta} a\right|_{y=x}$. Thus if $a \in S_{0,0}^{0, k}$, we have $\mathrm{Op},(a)=\mathrm{Op}_{i_{1}}(\tilde{a})$ with

$$
\tilde{a}=a-i \frac{\lambda_{2}}{\lambda_{1}} \eta \cdot \nabla_{\eta} a \bmod S_{0,0}^{0, k-1} .
$$

Let us now recall the construction of the parametrix in the elliptic zone. Recall (see e.g. [SV2]) that the operator $-\Delta_{e}$ has two sound speeds $c_{1}, c_{2}$ and the variety $\Sigma=\left\{\zeta \in T^{*} \Gamma ; c_{R}\|\zeta\|=1\right\}$ lies in the elliptic zone $\left\{\zeta \in T^{*} \Gamma ;\|\zeta\|>c_{1}^{-1}\right\},\|\cdot\|$ being the norm in $T^{*} \Gamma$. Let $\zeta^{0} \in T^{*} \Gamma$ with $\left\|\zeta^{0}\right\|>c_{1}^{-1}$ and from now on we assume that the space dimension is $n=3$. Let us pick local coordinates such that $\zeta^{0}=\left(0, \eta^{0}\right)$, the boundary is given locally by $x_{1}=0$ and the normal derivative at $x=0$ is given by $\partial / \partial x_{1}$. Then $x^{\prime}=\left(x_{2}, x_{3}\right)$ are local coordinates on $\Gamma$. Let $\chi_{\zeta^{0}}\left(x^{\prime}, \eta\right) \in C_{0}^{\infty}\left(T^{*} \Gamma\right)$ be a cut-off function equal to 1 near $\zeta^{0}$. If supp $\chi_{\zeta^{0}}$ is sufficiently small, one can construct a local FIO $H^{\zeta^{0}}$ with large parameter $\lambda \in \Lambda_{C_{1}, C_{2}}$ 
such that

$$
\left\{\begin{array}{l}
\left(\Delta_{e}+\lambda^{2}\right) H^{\zeta^{0}} f=K f \\
\left.H^{\zeta^{\circ}} f\right|_{I}=\mathrm{Op}_{\iota}\left(\chi_{\zeta^{0}}\right) f
\end{array}\right.
$$

where $K$ has kernel in $\tilde{C}^{\infty}$. The operator $H^{\zeta^{0}}$ is of the form

$$
H^{\zeta^{0}} f=\left(\frac{\lambda_{1}}{2 \pi}\right)^{2} \iint e^{i \lambda(\varphi(x, \eta)-y \cdot \eta)} h(x, \eta, \lambda) f(y, \lambda) d y d \eta .
$$

The phase function $\varphi$ solves the eikonal equation $(\nabla \varphi)^{2}=1,\left.\varphi\right|_{\Gamma}=x \cdot \eta$ to infinite order at $\Gamma$ and $\operatorname{Im} \varphi \geqq c x_{1}$ on $\operatorname{supp} \chi_{\zeta^{0}}$ with some $c>0$. This implies that $H^{\zeta^{0}} f=O\left(e^{-c \lambda_{1} x_{1}}\right)$. The matrix-valued amplitude $h$ is a solution of the corresponding transport equations and has the form $h=\sum_{j=0}^{\infty} h_{j}(x, \eta) \hat{\lambda}^{-J}$, with $h_{j}$ formal series in $x_{1}$. Set

$$
h^{(m)}=\sum_{j=0}^{m} \lambda^{-j} h_{-j}(x, \eta)
$$

and consider the operator $H_{m}^{\zeta^{0}}$ associated with $h^{(m)}$. Then $H_{m}^{\zeta^{0}}$ solves a problem similar to (15) with $K$ replaced by $K$ plus a FIO of order $-m$. Denote $N_{m}^{\zeta^{0}} f=$ $\left.\sum_{j=1}^{3} \boldsymbol{\sigma}_{J}\left(H_{m}^{\zeta 0} f\right) v_{j}\right|_{\Gamma}$, where $\boldsymbol{\sigma}_{j}={ }^{t}\left(\sigma_{1 j}, \sigma_{2,}, \sigma_{3 j}\right), \sigma_{i j}$ is the stress tensor. Then $N_{m}^{\zeta^{0}}$ is a $\Psi D O$ with large parameter $\lambda$ and symbol

$$
\sigma\left(N_{m}^{\zeta^{0}}\right)=\sum_{j=-1}^{m} \lambda^{-j} n_{-j}(x, \eta)
$$

The principal symbol is $\lambda n_{1}(x, \eta)=\lambda \chi_{\zeta^{0}}(x, \eta) \tilde{n}_{1}(x, \eta), \tilde{n}_{1}(x, \eta)$ being a Hermitian matrix with three distinct eigenvalues near $\Sigma$. One of the eigenvalues has simple zero at $\Sigma$, the other two are elliptic. Moreover, $\tilde{n}_{1}$ is elliptic everywhere in the elliptic zone outside $\Sigma$ (see also $[\mathrm{CP}, \mathrm{K}]$ ).

Thus for any $\zeta$ in a neighborhood of $\Sigma$ in the elliptic zone we constructed an operator $H_{m}^{\zeta}$ solving (15) provided that supp $\chi_{\zeta}$ is contained in a small neighborhood $U_{\zeta}$ of $\zeta$. Let $W_{1}$ be a bounded neighborhood of $\Sigma$ in the elliptic zone and let us pick a partition of unity $\left\{\chi_{\zeta^{j}}\right\}$ associated with $\left\{U_{\zeta}\right\}$ covering $W_{1}$ and supported in a slightly larger domain. Using this partition of unity, we construct a solution operator

$$
H_{m}(\hat{\lambda})=\sum_{j} H_{m}^{\zeta^{J}} \phi_{j}
$$

where $\phi_{j}(x)$ have small supports and $\phi_{j}(x)=1$ in a neighborhood of $\pi_{x}\left(\operatorname{supp} \chi_{\xi^{j}}\right)$. This operator solves

$$
\left\{\begin{array}{l}
\left(\Delta_{e}+\lambda^{2}\right) H_{m} f=K_{m} f \\
\left.H_{m} f\right|_{\Gamma}=f+Q f
\end{array}\right.
$$

provided that $\widehat{\mathrm{WF}}(f) \subset W_{1}$, where $K_{m}(\lambda)$ is a FIO with amplitude of order $-m$ and

$$
\|Q f\|_{H^{s}}=O\left(|\lambda|^{-\infty}\right), \quad \forall s \geqq 0 \text {. }
$$

Set

$$
N_{m} f=\left.\sum_{j=1}^{3} \boldsymbol{\sigma}_{J}\left(H_{m} f\right) v_{j}\right|_{\Gamma}
$$

Then $N_{m} \in L_{0,0}^{0,1}(\Gamma)$ and $\lambda^{m} N_{m}$ is holomorphic in $\lambda$. 
Now we are going to find a relationship between $N_{m}$ and the Neumann operator $\mathscr{N}$. Following $[\mathrm{G}]$, we set

$$
\tilde{H}_{m}(\lambda)=\chi H_{m}(\lambda)-S_{0}(\lambda)\left(\chi K_{m}(\lambda)+\left[\Delta_{e}, \chi\right] H_{m}(\lambda)\right),
$$

where $S_{0}(\lambda)$ is the free outgoing resolvent. Thus $\tilde{H}_{m}(\lambda) f$ solves the problem

$$
\left\{\begin{array}{l}
\left(\Delta_{e}+\lambda^{2}\right) \tilde{H}_{m} f=0, \\
\left.\tilde{H}_{m} f\right|_{\Gamma}=f+Q f+R_{m} f,
\end{array}\right.
$$

with

$$
\left\|R_{m}(\lambda)\right\|_{\mathscr{L}\left(H^{3 / 2}(\Gamma)\right)} \leqq C|\lambda|^{-m+1}
$$

provided that $\lambda \in \Lambda_{C_{1}, C_{2}}$ with $C_{1}$ small enough. If we denote the exact solution to (9) by $\mathscr{H}(\lambda) f$, we see that

$$
\mathscr{H}\left(f+Q f+R_{m} f\right)=\tilde{H}_{m} f .
$$

By differentiating this at the boundary, we get

$$
\mathscr{N}\left(f+Q f+R_{m} f\right)=N_{m} f+\tilde{R}_{m} f, \quad \widetilde{\mathrm{WF}}(f) \subset W_{1},
$$

where $\left\|\tilde{R}_{m}\right\|_{\mathscr{L}\left(H^{3 / 2}, H^{1 / 2}\right)}=O\left(|\lambda|^{-m+2}\right)$ and $Q f, R_{m}$ satisfy (19) and (22), respectively.

\section{Proof of the Main Result}

Let $\left\{\chi_{\llcorner}\right\}$be the partition of unity used to construct $H_{m}$ (see (17)). Choose an open set $W_{2} \subset T^{*} \Gamma$, such that $\Sigma \subset W_{2} \subset \subset W_{1}$ and pick $\chi_{0} \in C_{0}^{\infty}\left(T^{*} \Gamma\right)$, such that $\chi_{0}=1$ on $W_{2}$ and supp $\chi_{0} \subset W_{1}$. For each $\zeta^{j}$ let us define a local $\Psi$ DO using the special coordinates related to $\zeta^{j}$ by $A^{\zeta^{j}}=\mathrm{Op}_{i}\left(\chi_{0} \chi_{\zeta^{j}}\right)$ and set $A=\sum_{j} A^{\zeta^{j}} \phi_{j}$ (see (17)). Then $A \in L_{0,0}^{0,1}(\Gamma), A$ is an entire function of $\lambda$ and $\sigma_{p}(A)=1$ on $W_{2}, \sigma_{p}(A)=0$ outside $W_{1}$. Moreover, the symbol of $A$ in any local coordinates is supported in $W_{1}$. Since the symbol of $N_{m}$ has also compact support, we will extend $N_{m}$ as an operator elliptic outside $W_{1}$ with characteristic variety $\Sigma$. To this end fix an integer $m>0$ and set

$$
P(\lambda)=\lambda^{2 m-1} N_{2 m-1} A+i\left(\lambda^{2}-\Delta_{\Gamma}\right)^{m}(I-A),
$$

$\Delta_{\Gamma}$ being the Laplacian on $\Gamma$. Note first that $P$ is an analytic function of $\lambda$ with values in $\mathscr{L}\left(H^{s+2 m}, H^{s}\right)$. Secondly, let us mention that in any logarithmic region $\Lambda_{C_{1}, C_{2}}, P$ can be considered as a $\Psi D O$ with large parameter $\lambda_{1}=\operatorname{Re} \lambda$ and $P \in L_{0,0}^{2 m, 2 m}(\Gamma)$. We claim that $P$ is elliptic outside $\Sigma$. Indeed, for the principal symbol of $P$ we have

$$
\sigma_{p}(P)=\lambda^{2 m-1} \sigma_{p}(A) \sigma_{p}\left(N_{2 m-1}\right)+i \lambda_{1}^{2 m}\left(1-\sigma_{p}(A)\right)\left(\left(1+i \lambda_{2} / \lambda_{1}\right)^{2}+|\eta|_{x}^{2}\right)^{m},
$$

where $\sigma_{p}(A)$ is a function supported in $W_{1}$ and $|\eta|_{x}$ denotes the norm of the covector $(x, \eta)$. Note that here we consider $N_{2 m-1}, A$ as $\Psi$ DO-s with large parameter $\lambda_{1}$, not $\lambda$, and respectively $\sigma_{p}\left(N_{2 m-1}\right), \sigma_{p}(A)$ are the principal symbols of these operators obtained by using (14). In $W_{2} \backslash \Sigma$ the principal symbol $\sigma_{p}(P)$ is elliptic, because $\left.\sigma_{p}(A)\right|_{W_{2}}=1$ is elliptic. Outside $W_{1}, \sigma_{p}(P)=\lambda_{1}^{2 m}\left(\left(1+i \lambda_{2} / \lambda_{1}\right)^{2}+|\eta|_{x}^{2}\right)^{m}$ is elliptic as well, including at the infinite points of $\hat{T}^{*} \Gamma$. Finally, on $W_{1} \backslash W_{2}$ our 
claim follows from the fact that for any Hermitian elliptic matrix $B$ we have $|\alpha B x+i \beta x|^{2}=\alpha^{2}|B x|^{2}+\beta^{2}|x|^{2} \geqq c|x|^{2}$ provided that $\alpha+\beta=1$.

The next proposition establishes existence of "resonances" of $P$.

Proposition 2. There exist $\lambda_{j}$ and $f_{j} \in C^{\infty}(\Gamma), j=1,2, \ldots$, such that

a) $P\left(\lambda_{j}\right) f_{j}=0$,

b) $\left|\operatorname{Im} \lambda_{j}\right| \leqq C \ln \left|\lambda_{j}\right|$ and $\left|\lambda_{j}\right| \rightarrow \infty$, as $j \rightarrow \infty$,

c) $\left\|f_{j}\right\|_{H^{3 / 2}\left(I^{\prime}\right)}=1, \widehat{\mathrm{WF}}(f) \subset \Sigma$, where $f(x, \lambda):=f_{J}(x), \lambda=\lambda_{j}$.

To prove Proposition 2 we are going to apply the Phragmén-Lindelöf principle to $P^{-1}(\lambda)$. To this end we need the following two lemmae. First we prove an a priori exponential estimate of $P^{-1}(\lambda)$ similar to that in Lemma 2 (see also [SV2], Proposition 5.2).

Lemma 3. Assume that $P^{-1}(\lambda)$ has no poles in $\Lambda_{C_{1}, C_{2}}$ with some $C_{1}, C_{2}>0$. Then

$$
\left\|P^{-1}(\lambda)\right\|_{\mathscr{L}\left(L^{2}(\Gamma)\right)} \leqq C e^{C|\lambda|^{4}}, \quad \lambda \in \Lambda_{C_{1} / 2,2 C_{2}} .
$$

Proof. Let us rewrite $P(\lambda)$ in the form

$$
P(\lambda)=i\left(I-\Delta_{\Gamma}\right)^{m}(I+K(\lambda)),
$$

where $K(\lambda)=K_{1}(\lambda)+K_{2}(\lambda)$ with

$$
\begin{aligned}
& K_{1}(\lambda)=-\left[I+\left(\lambda^{2}-1\right)\left(I-\Delta_{\Gamma}\right)^{-1}\right]^{m} A-i\left(I-\Delta_{\Gamma}\right)^{-m} \lambda^{2 m-1} N_{2 m-1} A, \\
& K_{2}(\hat{\lambda})=\left[I+\left(\lambda^{2}-1\right)\left(I-\Delta_{\Gamma}\right)^{-1}\right]^{m}-I .
\end{aligned}
$$

Clearly, $K(\lambda)$ is an entire family of compact operators on $L^{2}(\Gamma)$. Moreover, the operator $K^{2}(\lambda)$ is of trace class and we can consider the entire function $h(\lambda)=$ $\operatorname{det}\left(I-K^{2}(\lambda)\right)$. As in the proof of Proposition 5.2 in [SV2] first we will prove the following a priori estimate

$$
\left\|P^{-1}(\lambda)\right\|_{\mathscr{L}\left(L^{2}(\Gamma)\right)} \leqq C e^{C|\lambda|^{4}}, \quad \lambda \in V,
$$

where $V=C \backslash \cup\left\{\lambda \in C ;\left|\lambda-z_{J}\right| \leqq\left|z_{J}\right|^{-4}\right\}, z_{j}$ being the zeros of $h$. To this end we will prove first that $h(\lambda)$ is of order 3 . We have

$$
\begin{aligned}
|h(\lambda)| & =\left|\operatorname{det}\left(I-K^{2}(\lambda)\right)\right| \leqq \prod_{j=1}^{\infty}\left(1+\mu_{j}\left(K^{2}(\lambda)\right)\right) \\
& \leqq \prod_{j=1}^{\infty}\left(1+\mu_{j}\left(\tilde{K}_{1}(\lambda)\right)\right)^{2} \prod_{j=1}^{\infty}\left(1+\mu_{j}\left(K_{2}^{2}(\lambda)\right)\right)^{2},
\end{aligned}
$$

where $\mu_{j}(K)$ denote the characteristic values of $K$ and $\tilde{K}_{1}=K_{1}^{2}+K_{1} K_{2}+K_{2} K_{1}$. Let us first estimate $\mu_{j}\left(\tilde{K}_{1}(\lambda)\right)$. Clearly,

$$
\mu_{J}\left(\tilde{K}_{1}(\lambda)\right) \leqq\left\|\tilde{K}_{1}(\lambda)\right\| \leqq C e^{C|i|}, \quad \forall \lambda \in \mathbf{C}, \forall j .
$$

On the other hand,

$$
\begin{aligned}
\mu_{J}\left(\tilde{K}_{1}(\lambda)\right) & \leqq \mu_{\left[\frac{l}{2}\right]}\left(K_{1}^{2}+K_{1} K_{2}\right)+\mu_{\left[\frac{l}{2}\right]}\left(K_{1} K_{2}\right) \\
& \leqq\left\|K_{1}+K_{2}\right\| \mu_{\left[\frac{l}{2}\right]}\left(K_{1}\right)+\left\|K_{2}\right\| \mu_{\left[\frac{J}{2}\right]}\left(K_{1}\right) \\
& \leqq C e^{C|\hat{\lambda}|} \mu_{\left[\frac{j}{2}\right]}(A) .
\end{aligned}
$$


Let us recall that $A$ is a finite sum of operators $A^{\zeta} \phi$ with kernels of the kind

$$
\left(\frac{\lambda}{2 \pi}\right)^{2} \chi(x, \eta) e^{i \lambda(x-y) \cdot \eta} \phi(y)
$$

where $\chi$ is a cut-off function supported near $\zeta$ in the elliptic zone, $\phi \in C_{0}^{\infty}$ and $\phi(y)=1$ in a neighborhood of $\pi_{x}(\operatorname{supp} \chi)$. Set $M:=\pi_{\eta}(\operatorname{supp} \chi)$. We have $A^{\zeta}=$ $A_{1} A_{2}$, where $A_{1}: L^{2}(M) \rightarrow L^{2}(\Gamma), A_{2}: L^{2}(\Gamma) \rightarrow L^{2}(M)$ have kernels $(\lambda / 2 \pi)^{2} \chi(x, \eta)$ $e^{i j x \cdot \eta}$ and $e^{-i \lambda y \cdot \eta} \phi(y)$, respectively. For the kernel of $A_{2}$ we have

$$
\sup _{y \in \operatorname{supp} \phi, \eta \in M}\left|\left(I-\Delta_{\eta}\right)^{k} e^{-l i y \cdot \eta} \phi(y)\right| \leqq C e^{C|\lambda|}\left((C|\lambda|)^{2 k}+(2 k)^{2 k}\right) .
$$

Therefore, we get for any $k \geqq 0$ and for any $j>0$,

$$
\mu_{j}\left(A_{2}\right) \leqq \mu_{j}\left(\left(I-\Delta_{\eta}\right)^{-k}\right)\left\|\left(I-\Delta_{\eta}\right)^{k} A_{2}\right\| \leqq C j^{-k} e^{C|\lambda|}\left((C|\lambda|)^{2 k}+(2 k)^{2 k}\right) .
$$

Taking $k=[|\lambda| / 2], j \geqq C(q)|\lambda|^{2}$ gives

$$
\mu_{j}\left(A_{2}\right) \leqq j^{-2} e^{-q|\lambda|} \quad \text { for any } q>0 \quad \text { and } \quad j \geqq C(q)|\lambda|^{2} \text {. }
$$

By (30) and the estimate $\left\|A_{1}\right\| \leqq C e^{C|\lambda|}$ we get the same type of estimate for $A^{\zeta}$, and hence for $A$. Thus, choosing $q$ properly, in view of (29) we obtain

$$
\mu_{j}\left(\tilde{K}_{1}(\lambda)\right) \leqq C j^{-2} \text { for } j \geqq C^{\prime}|\lambda|^{2} .
$$

Combining (28) and (31) yields

$$
\prod_{j=1}^{\infty}\left(1+\mu_{J}\left(\tilde{K}_{1}(\lambda)\right)\right)^{2} \leqq \prod_{j \leqq C^{\prime}|\lambda|^{2}}\left(C e^{C|\lambda|}\right)^{2} \prod_{j>C^{\prime}|\lambda|^{2}}\left(1+C^{\prime} j^{-2}\right)^{2} \leqq C e^{C|\lambda|^{3}}
$$

It remains to estimate $\mu_{j}\left(K_{2}^{2}\right)$. We have

$$
\mu_{j}\left(K_{2}^{2}\right) \leqq \mu_{\left[\frac{J}{2}\right]}^{2}\left(K_{2}\right) .
$$

On the other hand,

$$
K_{2}=\sum_{p=1}^{m}\left(\begin{array}{c}
m \\
p
\end{array}\right)\left(\lambda^{2}-1\right)^{p}\left(I-\Delta_{\Gamma}\right)^{-p},
$$

thus, setting $\langle\lambda\rangle:=\left(1+|\lambda|^{2}\right)^{1 / 2}$, we get

$$
\begin{aligned}
\mu_{J}\left(K_{2}\right) & \leqq \sum_{p=1}^{m}\left(\begin{array}{c}
m \\
p
\end{array}\right)\langle\lambda\rangle^{2 p} \mu_{\left[\frac{j}{m}\right]}\left(\left(I-\Delta_{\Gamma}\right)^{-p}\right) \leqq \sum_{p=1}^{m}\left(\begin{array}{c}
m \\
p
\end{array}\right)\langle\lambda\rangle^{2 p}\left(\frac{c j}{m}\right)^{-p} \\
& \leqq C\langle\lambda\rangle^{2} j^{-1} \quad \text { for } j \geqq\langle\lambda\rangle^{2} / 2
\end{aligned}
$$

Thus we get from (33) and the estimate above

$$
\mu_{j}\left(K_{2}^{2}\right) \leqq C\langle\lambda\rangle^{4} j^{-2} \text { for } j \geqq\langle\lambda\rangle^{2} .
$$


Using (34) we deduce

$$
\begin{aligned}
\prod_{j=1}^{\infty}\left(1+\mu_{j}\left(K_{2}^{2}(\lambda)\right)\right) & \leqq \prod_{j \leqq\langle\lambda\rangle^{2}}\left[C\langle\lambda\rangle^{2 m}\right] \prod_{j>\langle\lambda\rangle^{2}}\left(1+C\langle\lambda\rangle^{4} j^{-2}\right) \\
& \leqq \exp \left[C\langle\lambda\rangle^{2} \ln \langle\lambda\rangle\right] \exp \left[C\langle\lambda\rangle^{4} \sum_{j>\langle\lambda\rangle^{2}} j^{-2}\right] \\
& \leqq \exp \left[C\langle\lambda\rangle^{2} \ln \langle\lambda\rangle\right] \exp \left[C\langle\lambda\rangle^{2}\right] \leqq C e^{C|\lambda|^{3}}
\end{aligned}
$$

Now (32) and (35) together imply

$$
|h(\lambda)| \leqq \prod_{j=1}^{\infty}\left(1+\mu_{j}\left(K^{2}(\lambda)\right)\right) \leqq C e^{C|\lambda|^{3}}, \quad \lambda \in \mathbf{C} .
$$

We will complete the proof of the lemma as in [SV2]. By [Ti, Ch. VIII] we conclude from (36) that

$$
\left|h^{-1}(\lambda)\right| \leqq C e^{C|\lambda|^{4}}, \quad \lambda \in V .
$$

On the other hand, we have (see e.g. [GK, Theorem 5.1])

$$
\left|\operatorname{det}\left(I-K^{2}(\lambda)\right)\right| \cdot\left\|\left(I-K^{2}(\lambda)\right)^{-1}\right\| \leqq \prod_{j=1}^{\infty}\left(1+\mu_{j}\left(K^{2}(\lambda)\right)\right) \leqq C e^{C|\lambda|^{3}} .
$$

By (37) and (38) we obtain

$$
\left\|\left(I-K^{2}(\lambda)\right)^{-1}\right\| \leqq C e^{C|\lambda|^{4}}, \quad \lambda \in V,
$$

which implies immediately (26). As in the proof of Lemma 2 if we assume that $P^{-1}$ is free of poles in some logarithmic domain, we will get that (26) holds in a slightly shrunken domain.

Denote $l_{ \pm}=\left\{\lambda \in C ; \operatorname{Re} \lambda \geqq C_{2}, \operatorname{Im} \lambda= \pm C_{1} \ln (\operatorname{Re} \lambda)\right\}$. Let us assume that $C_{2}>1$, so that $\ln (\operatorname{Re} \lambda)>0$.

Lemma 4. For any $C_{1}>0$ there exists $C_{2}>1$, such that the operator $P(\lambda)$ is invertible on $l_{ \pm}$and

$$
\left\|P^{-1}(\lambda)\right\|_{\mathscr{L}\left(L^{2}(\Gamma)\right)} \leqq \frac{C}{\ln |\lambda|}|\lambda|^{-2 m+1}, \quad \lambda \in l_{ \pm} .
$$

Proof. Let $\zeta \notin W_{2}$ and $\chi \in C_{0}^{\infty}\left(T^{*} \Gamma\right)$ be a cut-off function with sufficiently small support in $T^{*} \Gamma \backslash W_{2}$. Since $P$ is a $\Psi$ DO with large parameter $\lambda_{1}$ elliptic outside $W_{2}$, we get

$$
\left\|\mathrm{Op}_{\lambda_{1}}(\chi) f\right\| \leqq \frac{C}{|\lambda|^{2 m}}\|P f\|+C_{N}|\lambda|^{-N}\|f\|
$$

for any $N>0$ and $\lambda \in l_{ \pm}$, where $\mathrm{Op}_{\lambda_{1}}(\chi)$ is the $\Psi$ DO with symbol $\chi$ written in the special coordinates related to $\zeta$. The same estimate holds if $\chi$ is supported near the infinite points in $\hat{T}^{*} \Gamma$, i.e. for $\chi=\chi^{\prime}(x) \chi^{\prime \prime}(\eta)$, where supp $\chi^{\prime}$ is close to a point $x^{0} \in \Gamma$, while supp $\chi_{2} \subset\left\{\eta ; c_{R}|\eta|>2\right\}$.

Let us now choose $\zeta \in W_{1}$ and pick $\chi \in C_{0}^{\infty}\left(T^{*} \Gamma\right)$ supported in $W_{1}$, such that $\chi=1$ in a neighborhood of $\zeta$. The principal symbol of $P$ considered as a $\Psi$ DO with large parameter $\lambda \in l_{ \pm}$is $\lambda^{2 m} \tilde{n}_{1}(x, \eta)$ (see Sect. 3 ). In a neighborhood $U_{\zeta}$ of supp $\chi$ we have

$$
T^{*} \tilde{n}_{1} T=\operatorname{diag}\left(c_{R}^{2}|\eta|_{x}^{2}-1,1,1\right) S
$$


where $S=\operatorname{diag}\left(a_{1}^{\prime}, a_{2}, a_{3}\right)$ is elliptic and $a_{1}=\left(c_{R}^{2}|\eta|_{x}^{2}-1\right) a_{1}^{\prime}, a_{2}, a_{3}$ are the eigenvalues of $\tilde{n}_{1}$. Here $T$ is a unitary matrix. Let us now consider $\mathrm{Op}_{\lambda}\left(c_{R}^{2}|\eta|_{x}^{2}-1\right)$ as a $\Psi$ DO with large parameter $\lambda_{1}$. From (14) we deduce that the principal symbol of the latter reads

$$
\frac{1}{\lambda^{2}}\left(\lambda_{1}^{2} c_{R}^{2}|\eta|_{x}^{2}-\lambda_{1}^{2}-2 i \lambda_{1} \lambda_{2}\right)
$$

Therefore, modulo $S_{0,0}^{0,-1}$ this operator coincides with

$$
\frac{1}{\lambda^{2}}\left(-\Delta_{\Gamma}-\lambda_{1}^{2}-2 i \lambda_{1} \lambda_{2}\right)
$$

Observe that for any $g$ and $C_{2}$ sufficiently large

$$
\left\|\frac{1}{\lambda^{2}}\left(-\Delta_{\Gamma}-\lambda_{1}^{2}-2 i \lambda_{1} \lambda_{2}\right) g\right\| \geqq \frac{\left|\lambda_{2}\right|}{|\lambda|}\|g\|=C_{1} \frac{\ln \lambda_{1}}{|\lambda|}\|g\| .
$$

This inequality together with (40) shows that for any $N>0$ we have

$$
\left\|\mathrm{Op}_{\lambda}(\chi) f\right\| \leqq \frac{C}{|\lambda|^{2 m-1} \ln |\lambda|}\|P f\|+C_{N}|\lambda|^{-N}\|f\| .
$$

Choose $\chi_{1} \in C_{0}^{\infty}\left(T^{*} \Gamma\right)$, such that supp $\chi_{1} \subset\{\zeta ; \chi(\zeta)=1\}$. Then one easily gets

By (39), (41),

$$
\left\|\mathrm{Op}_{i_{1}}\left(\chi_{1}\right) f\right\| \leqq \frac{C}{|\lambda|^{2 m-1} \ln |\lambda|}\|P f\|+C_{N}|\lambda|^{-N}\|f\| .
$$

$$
\|f\| \leqq \frac{C}{|\lambda|^{2 m-1} \ln |\lambda|}\|P f\|
$$

for any $f$ and $|\lambda|$ sufficiently large. Since we can prove the same type of estimate for $P^{*}$, we get that $P^{-1}(\lambda)$ exists for $\lambda \in l_{ \pm}$sufficiently large and satisfies the desired estimate.

Proof of Proposition 2. Assume now that there is a finite number of poles of $P^{-1}$ in some logarithmic domain $\Lambda_{C_{1}, C_{2}}$. Taking $C_{2}$ sufficiently large we can assume that $\Lambda_{C_{1}}, C_{2}$ is free of poles and Lemma 4 holds. Let us apply the PhragménLindelöf principle to the function $\lambda^{2 m-1}(\log \lambda) P^{-1}(\lambda)$ in $\Lambda_{C_{1} / 2,2 C_{2}}$. By Lemma 3 it satisfies the a priori exponential estimate in $\Lambda_{C_{1} / 2,2 C_{2}}$, while by Lemma 4 it is uniformly bounded on the boundary. Therefore, it is uniformly bounded in $\Lambda_{C_{1} / 2,2 C_{2}}$ as well, i.e.

$$
\left\|P^{-1}(\lambda)\right\|_{\mathscr{L}\left(L^{2}(\Gamma)\right)} \leqq \frac{C}{|\lambda|^{2 m-1} \ln |\lambda|}, \quad \lambda \in \Lambda_{C_{1} / 2,2 C_{2}} .
$$

The final step of the proof of Proposition 2 is to show that for real $\lambda$, (42) leads to contradiction for real $\lambda$. We will do this in exactly the same way as in [SV2]. Let $\left\{\mu_{j}^{2}\right\}$ be the eigenvalues of $-c_{R}^{2} \Delta_{\Gamma}$ and denote by $\varphi_{l},\left\|\varphi_{j}\right\|=1$ the corresponding eigenfunctions. Fix $\zeta^{0} \in \Sigma$ and let $\chi$ be supported in a small neighborhood $U$ of $\zeta^{0}$ in the elliptic region. Let $\Pi(x, \eta),(x, \eta) \in U$ be the projection onto the eigenspace corresponding to the first eigenvalue $a_{1}=\left(c_{R}^{2}|\eta|_{x}^{2}-1\right) a_{1}^{\prime}$. Set

$$
f_{k}\left(\cdot, \mu_{J}\right)=\mathrm{Op}_{\mu_{j}}(\chi \Pi) e_{k} \varphi_{j}
$$

$\left\{e_{k}\right\}_{k=1}^{3}$ being the standard base in $\mathbf{R}^{3}$. Denote $\Theta=\left\{\mu_{\jmath}\right\}_{\jmath=1}^{\infty}$ and $f_{k}(x, \lambda)=f_{k}\left(x, \mu_{J}\right)$, $\varphi(x, \lambda)=\varphi_{J}(x)$ for $\lambda \in \Theta$. Consider all $\Psi$ DO-s below as $\Psi$ DO-s with large parameter $\lambda \in \Theta$. Then

$$
P f_{k}=G e_{k} \varphi,
$$


where $G \in L_{0,0}^{0,2 m}(\Gamma), \sigma_{p}(G)=\lambda^{2 m}\left(c_{R}^{2}|\eta|_{x}^{2}-1\right) a_{1}^{\prime} \chi \Pi$. Since the principal symbol of $-c_{R}^{2} \Delta_{\Gamma}-\lambda^{2}$ is $\lambda^{2}\left(c_{R}^{2}|\eta|_{x}^{2}-1\right)$, we have

$$
P f_{k}=\lambda^{2 m-2} \mathrm{Op}\left(\chi a_{1}^{\prime} \Pi\right)\left(-c_{R}^{2} \Delta_{\Gamma}-\lambda^{2}\right) e_{k} \varphi+B e_{k} \varphi=B e_{k} \varphi,
$$

where $B \in L_{0,0}^{0,2 m-1}(\Gamma)$. Thus

$$
\left\|P f_{k}\right\| \leqq C \lambda^{2 m-1} \text { for } k=1,2,3 ; \lambda \in \Theta .
$$

According to (42), (43),

$$
\left\|\mathrm{Op}(\chi \Pi) e_{k} \varphi\right\| \leqq \frac{C}{\ln \lambda} \text { for } k=1,2,3 ; \lambda \in \Theta .
$$

Since the projection $\Pi(\zeta)$ is well defined and does not vanish near $\Sigma$, we have that $\sum\left|\Pi_{l j}\right|^{2}$ is elliptic in $U$ provided that $U$ is sufficiently close to $\Sigma$. Thus from (47) we deduce that

$$
\left\|\mathrm{Op}\left(\chi^{\prime} \chi^{\prime \prime}\right) \varphi\right\| \leqq \frac{C}{\ln \lambda},
$$

where $\chi^{\prime}=\chi^{\prime}(x), \chi^{\prime \prime}=\chi^{\prime \prime}(\eta)$ and $\chi^{\prime}(x)=1, \chi^{\prime \prime}(\eta)=1$ for $(x, \eta)$ close to $\zeta^{0}$, supp $\chi^{\prime} \chi^{\prime \prime} \subset\{\chi=1\}$. On the other hand, $\left(-c_{R}^{2} \Delta_{\Gamma}-\lambda^{2}\right) \varphi=0$ and $-c_{R}^{2} \Delta_{\Gamma}-\lambda^{2}$ is a $\Psi$ DO on $\Gamma$ in $L_{0,0}^{2.2}(\Gamma)$ with principal symbol $\lambda^{2}\left(c_{R}^{2}|\eta|_{x}^{2}-1\right)$ elliptic outside $\Sigma$. Therefore, $\widehat{\operatorname{WF}}(\varphi) \subset \Sigma$. Hence,

$$
\left\|\mathrm{Op}\left(\chi^{\prime}\left(1-\chi^{\prime \prime}\right)\right) \varphi\right\| \leqq C_{N} \lambda^{-N}, \quad \forall N>0 .
$$

Combining (48) and (49) we get

$$
\left\|\chi^{\prime} \varphi\right\| \leqq \frac{C}{\ln \lambda}, \quad \lambda \in \Theta
$$

for any cut-off function $\chi^{\prime}$, such that $\chi^{\prime}=1$ near $x^{0}=\pi_{x}\left(\zeta^{0}\right)$ and $\operatorname{supp} \chi^{\prime}$ is sufficiently small. Since $\zeta^{0} \in \Sigma$ was arbitrary, we get $\|\varphi\| \leqq C / \ln i$ which contradicts the fact that $\|\varphi\|=1$.

Thus, there exists a sequence $\{\lambda$,$\} of poles of P^{-1}(\lambda)$ satisfying (b). Going back to the representation (25) we conclude by the Fredholm alternative that for any pole $\lambda_{j}$ there is a function $f_{j} \neq 0$ such that $P\left(\lambda_{j}\right) f_{j}=0$. Since $P$, considered as a $\Psi$ DO with large parameter $\lambda_{1}$ (with $\lambda$ satisfying (b)) is elliptic outside $\Sigma$, we get (c).

Next we show that Proposition 2 implies existence of asymptotic zeros of $N_{2 m-1}$.

Proposition 3. Let $\lambda_{j}, f_{j}, j=1,2, \ldots$ be as in Proposition 2. Then

(a) $N_{2 m-1}\left(\lambda_{j}\right) f_{J}=O\left(\left|\lambda_{j}\right|^{-\infty}\right)$,

(b) $\left|\operatorname{Im} \lambda_{j}\right| \leqq C\left|\lambda_{j}\right|^{-2 m+2}$ with some $C>0$.

Proof. It follows from Proposition 2 (c) that $(I-A) f_{j}=O\left(\left|\lambda_{j}\right|^{-\infty}\right)$, which in view of (24) yields (a). To prove (b), set $f(x, \lambda)=f_{j}(x), \lambda \in \Theta:=\left\{\lambda_{j}\right\}_{j=1}^{\infty}$ and recall that $H_{2 m-1}$ solves (18) with $2 m-1$ instead of $m$. Arguing as in [SV2], we get that

$$
\left(\Delta_{e}+\lambda^{2}\right) \phi H_{2 m-1} f=\left[\Delta_{e}, \phi\right] H_{2 m-1} f+\phi K_{2 m-1} f,
$$

where $\phi \in C_{0}^{\infty}, \phi=1$ near $\Gamma$. Multiply (50) by $\phi H_{2 m-1} f$ and integrate by parts. Using (18), we get that

$$
\operatorname{Im} \lambda^{2} \leqq \frac{\left\|\left[\Delta_{e}, \phi\right] H_{2 m-1} f\right\|+C|\lambda|^{-2 m+1}}{\left\|\phi H_{2 m-1} f\right\|} .
$$


Now, we use the facts that $\left[\Delta_{e}, \phi\right]$ is a first order differential operator with compactly supported coefficients vanishing near $\Gamma$ and that the parametrix in the elliptic zone decays exponentially in $|\lambda| x_{1}$. This implies

$$
\left\|\left[\Delta_{e}, \phi\right] H_{2 m-1} f\right\| \leqq C e^{-\gamma|\lambda|}\|f\| \leqq C e^{-\gamma|\hat{\lambda}|}
$$

with some $\gamma>0$ (recall that $\|f\|_{H^{3 / 2}}=1$ ). On the other hand, by trace theorem, in view of (17) and using the fact that the operator $\Delta_{e}$ with Dirichlet boundary conditions is coercive, we have

$$
\begin{aligned}
\|f+Q f\|_{H^{3 / 2}} & \leqq C\left\|\phi H_{2 m-1} f\right\|_{H^{2}} \leqq C^{\prime}\left(\left\|\Delta_{e} \phi H_{2 m-1} f\right\|+\left\|\phi H_{2 m-1} f\right\|\right) \\
& \leqq C^{\prime \prime}|\lambda|^{2}\left\|\phi H_{2 m-1} f\right\|+C^{\prime \prime}|\lambda|^{-2 m+1}
\end{aligned}
$$

which gives for large $\lambda \in \Theta$

$$
1 \leqq 2 C^{\prime \prime}|\lambda|^{2}\left\|\phi H_{2 m-1} f\right\|
$$

Combining the above estimates implies (b) at once.

We are ready now to conclude the proof of Theorem 1. Let us see first that for any integer $N \geqq 1$ there are infinitely many resonances in $\{\lambda \in \mathbf{C}$ : $\left.\operatorname{Im} \lambda \leqq|\lambda|^{-N},|\operatorname{Re} \lambda| \geqq 1\right\}$. Assume the contrary, i.e. $R_{\gamma}(\lambda)$ is holomorphic in $\left\{\lambda \in \overline{\mathbf{C}}: \operatorname{Im} \lambda \leqq|\lambda|^{-\bar{N}},|\operatorname{Re} \lambda| \geqq C_{0}\right\}$ for some constant $C_{0}>0$. Choose $m$ so that $2 m-3>N+9$. Let $f_{j}, \lambda_{j}$ be as in Propositions 2 and 3 , and set $f(x, \lambda)=f_{j}(x)$, $\lambda \in \Theta:=\left\{\lambda_{J}\right\}_{J=1}^{\infty}$. In view of Proposition 2(c) we can use (23) to obtain

$$
N_{2 m-1} f+\tilde{R}_{2 m-1} f=\mathscr{N}\left(f+Q f+R_{2 m-1} f\right), \quad \lambda \in \Theta .
$$

Since $\Theta \cap\left\{\lambda ;|\operatorname{Re} \lambda| \geqq C_{1}\right\} \subset\left\{\lambda \in \mathbf{C}:|\operatorname{Im} \lambda| \leqq|\lambda|^{-N-6},|\operatorname{Re} \lambda| \geqq C_{1}\right\}$ for $C_{1}$ large enough, by (10) we obtain

$$
\left\|f+Q f+R_{2 m-1} f\right\|_{H^{3 / 2}} \leqq C|\lambda|^{N+9}\left\|N_{2 m-1} f+\tilde{R}_{2 m-1} f\right\|_{H^{1 / 2}} \leqq C^{\prime}|\lambda|^{N+9-(2 m-3)} .
$$

On the other hand, in view of (19), (22),

$$
1=\|f\|_{H^{3 / 2}} \leqq\left\|f+Q f+R_{2 m-1} f\right\|_{H^{3 / 2}}+C|\lambda|^{-1}
$$

therefore we get a contradiction for large $\lambda=\lambda_{j}$.

We will now choose our sequence of resonances by induction. Assume that we have already chosen $\lambda_{1}, \ldots, \lambda_{k-1}$. It follows from the above analysis that there exists a resonance $\lambda_{k}$ satisfying $\left|\lambda_{k}\right|>\left|\lambda_{k-1}\right|+1$ and

$$
0<\operatorname{Im} \lambda_{k} \leqq\left|\lambda_{k}\right|^{-k}
$$

Thus we have an infinite sequence of different resonances $\left\{\lambda_{k}\right\}$ satisfying (51) for each $k \geqq 1$. It is easy to see now that

$$
0<\operatorname{Im} \lambda_{k} \leqq C_{N}\left|\lambda_{k}\right|^{-N}, \quad \forall k,
$$

for any integer $N \geqq 1$ with $C_{N}=\left|\lambda_{N}\right|^{N}$. Indeed, for $k \geqq N$ (52) follows from (51) at once, while for $k \leqq N$ we have

$$
\operatorname{Im} \lambda_{k} \leqq\left|\lambda_{k}\right|^{-k} \leqq\left|\lambda_{N}\right|^{N}\left|\lambda_{k}\right|^{-N}
$$

which completes the proof of (52), and hence of Theorem 1 . 


\section{References}

[CP] Cardoso, F., Popov, G.: Rayleigh quasimodes in linear elasticity. Comm. P.D.E. 17, 1327-1367 (1992)

[G] Gérard, C.: Asymptotique des poles de la matrice de scattering pour deux obstacles strictement convex. Bull. Soc. Math. France, Mémoire n. 31, 116, 1988

[GK] Gohberg, I., Krein, M.: Introduction to the theory of linear non-selfadjoint operators. Providence, RI: AMS, 1969

[I1] Ikawa, M.: On the poles of the scattering matrix for two strictly convex obstacles. J. Math. Kyoto Univ. 23-1, 127-194 (1983)

[12] Ikawa, M.: Precise information on the poles of the scattering matrix for two strictly convex obstacles. J. Math. Kyoto Univ. 27-1, 69-102 (1987)

[13] Ikawa, M.: Trapping obstacles with a sequence of poles of the scattering matrix converging to the real axis. Osaka J. Math. 22, 657-689 (1985)

[K] Kawashita, M.: On the local-energy decay property for the elastic wave equation with the Neumann boundary conditions. Duke Math. J. 67, 333-351 (1992)

[L] Lazutkin, V.: Asymptotics of the eigenvalues of the Laplacian and quasimodes. Math. USSR Izvestija 7, 439-466 (1973)

[P] Popov, G.: Quasimodes for the Laplace operator and glancing hypersurfaces. In: M. Beals, R. Melrose, J. Rauch (eds.): Proceeding of Conference on Microlocal Analysis and nonlinear waves, Minnesota 1989, Berlin-Heidelberg-New York: Springer, 1991

[SV1] Stefanov, P., Vodev, G.: Distribution of resonances for the Neumann problem in linear elasticity outside a ball. Ann. Inst. H. Poincaré (Physique Théorique) 60, 303-321 (1994)

[SV2] Stefanov, P., Vodev, G.: Distribution of resonances for the Neumann problem in linear elasticity outside a strictly convex body. Duke Math. J. 78, 677-714 (1995)

[T] Taylor, M.: Rayleigh waves in linear elasticity as a propagation of singularities phenomenon. In: Proc. Conf. on P.D.E. and Geometry, New York: Marcel Dekker, 1979, pp. 273-291

[Ti] Titchmarsh, E.C.: The Theory of Functions. Oxford: Oxford Univ. Press, 1968

[Y] Yamamoto, K.: Singularities of solutions to the boundary value problems for elastic and Maxwell's equations. Japan J. Math. 14, 119-163 (1988)

Communicated by B. Simon 
